\title{
Desenlaces clínicos en nefritis lúpica. Reporte de una serie de casos del Hospital Militar Central de Bogotá.
}

\author{
John Alejandro Camargo ${ }^{1}$, Jorge Armando Pulido ${ }^{1}$, Juan Guillermo Vargas ${ }^{1,2}$, \\ Roberto D Achiardi ${ }^{2}$, Jorge Enrique Echeverri ${ }^{1,2}$ \\ ${ }^{1}$ Universidad Militar Nueva Granada, Bogotá, Colombia
2 Servicio de nefrología RTS - Hospital Militar Central, Bogotá, Colombia
}

\section{Resumen}

La presente serie retrospectiva de casos, pretende describir las características y desenlaces clínicos de los pacientes del Hospital Militar Central de Bogotá, con nefritis lúpica proliferativa. Es frecuente encontrar al momento del diagnóstico proteinuria, $37 \%$ de ellas en rango nefrótico y alteraciones en el uroanálisis. Describimos los resultados de las principales variables hematológicas e inmunológicas. No encontramos diferencias entre los tipos de inducción ni en los desenlaces como: porcentaje de remisión, disminución de creatinina y reducción de proteinuria.

Palabras clave: nefritis lúpica, inmunosupresión.

\section{Clinical Outcomes in Lupus Nephritis. Report of a series of cases in the Central Military Hospital in Bogotá.}

\begin{abstract}
This retrospective case series aims to describe the characteristics and clinical outcomes of patients in the Central Military Hospital in Bogotá, with proliferative lupus nephritis. It is commonly found at the time of diagnosis proteinuria, $37 \%$ of them in the nephrotic range, and changes in the urinalysis. We describe the results of the main hematological and immunological variables. We found no differences between the types of induction and outcomes such as the rate of remission, decreased creatinine and proteinuria reduction.
\end{abstract}

Keywords: lupus nephritis, immunosuppression.

\section{Introducción}

L

a nefritis lúpica (NL) es una de las manifestaciones más severas del lupus eritematoso sistémico (LES) y se presenta entre el $30-50 \%$ de los pacientes ${ }^{1}$. Sus manifestaciones clínicas dependen del grado de compromiso a nivel renal, describiéndose proteinuria en $90 \%$, hematuria en $80 \%$ y 
deterioro de la función renal entre 40 y $80 \%$ de los casos. Existe correlación clínica con la clase histopatológica, considerándose las clases III, IV y V de alta actividad serológica. Se reconoció en NL la clase IV como la de mayor actividad y peor pronóstico, la cual se presenta entre el 35 y el $60 \%$ de las biopsias renales. Se describen en la literatura distintos esquemas de inducción con diferentes desenlaces.

En los casos de tratamiento con ciclofosfamida, el esquema NIH reporta remisión de $85 \%$ y el Euro-lupus remisión del $71 \% \%^{2,3}$. La inducción con micofenolato mofetil reporta remisión del $56.2 \%{ }^{4}$. No hay consenso sobre la superioridad de un esquema de inducción sobre otro al evaluar la población en Latinoamérica ${ }^{5}$. Consideramos conveniente identificar la severidad de la NL y los desenlaces con las diferentes terapias de inducción, en los pacientes atendidos en nuestra institución.

\section{Objetivos e hipótesis}

Describir las características y desenlaces clínicos de los pacientes del Hospital Militar Central de Bogotá, con diagnóstico de nefritis lúpica.

\section{Diseño}

Estudio observacional, descriptivo, tipo serie de casos.

\section{Materiales y métodos}

Estudio descriptivo, retrospectivo en el que se incluyeron los pacientes del Hospital Militar Central de Bogotá, que consultaron los servicios de nefrología y reumatología, en el período comprendido entre el 1 de enero de 2009 y el 1 de enero de 2014, y que cumplieron con la definición y criterios clínico patológicos de NL de acuerdo con los criterios de la Guía de Práctica Clínica de Glomerulonefritis KDIGOISN/RPS ${ }^{1}$.

La sospecha diagnóstica se confirmó mediante patología renal. Se excluyeron los pacientes con diagnóstico no confirmado de la enfermedad. Se recolectó información demográfica, clínica y de laboratorio, mediante la revisión de las historias clínicas. Para este estudio no se requirió consentimiento informado por escrito, por tratarse de una investigación sin riesgo, según la Resolución 8430 de 1993. El estudio cumplió con los principios básicos de investigación proclamados en la Declaración de Helsinki de la Asociación Médica Mundial. Los datos fueron almacenados y analizados en el programa SPSS versión 18. Las variables cuantitativas se describen mediante medidas de tendencia central y las cualitativas como frecuencias relativas.

\section{Resultados}

Durante el período descrito se confirmó el diagnóstico de NL en 21 pacientes, de los cuales 2 no cumplieron con los criterios de inclusión al no completar el seguimiento, uno presentaba NL clase I y 2 no tenían disponible el reporte de patología renal. La población del estudio está constituida por 19 pacientes: 11 mujeres $(57.1 \%)$, con una edad promedio de 34.87 años (18 - 67 años) y el 100\% de los pacientes eran mestizos. El tiempo promedio de seguimiento fue de 95.14 meses $(3-152$ meses) (Tabla 1$)$.

La proporción de síntomas no renales encontrados, según la escala de BILAG ${ }^{6}$, al momento del diagnóstico fue: generales $25 \%$; cardiorespiratorios $31 \%$; gastrointestinales $0 \%$; hematológicos $13 \%$; mucocutáneos 44\%; musculoesqueléticos 38\% y neurológicos $0 \%$. En 2 pacientes se presentaron únicamente síntomas no renales de LES. La distribución por tipo de NL (de acuerdo con los criterios ISN/RPS) fue: clase I: 1 paciente, clase II: 3 pacientes, clase III: 5 pacientes, clase IV: 8 pacientes, no disponible (ND): 2 pacientes.

Con respecto a las variables basales de laboratorio, la creatinina promedio fue de $1.15 \mathrm{mg} / \mathrm{dL}$ (0.51-4.99 $\mathrm{mg} / \mathrm{dL})$, la tasa de filtración glomerular estimada (FGe) (CKD-EPI) promedio fue de 93.39 $\mathrm{ml} / \mathrm{min} / 1.73 \mathrm{~m}^{2}\left(10.97-148.09 \mathrm{ml} / \mathrm{min} / 1.73 \mathrm{~m}^{2}\right)$ y el BUN promedio fue de $18.71 \mathrm{mg} / \mathrm{dL}(7.5-45.5 \mathrm{mg} /$ dL) (Tabla 2).

En el parcial de orina basal se encontró que la densidad urinaria promedio fue de 1.018 (1.009-1.031), $\mathrm{pH}$ promedio fue de 5.8 (5-6.5), proteinuria en el $79 \%$, hematuria en el $84 \%$ y leucocituria en el $37 \%$ 


\begin{tabular}{|c|c|c|c|c|c|c|c|}
\hline \multicolumn{8}{|c|}{ 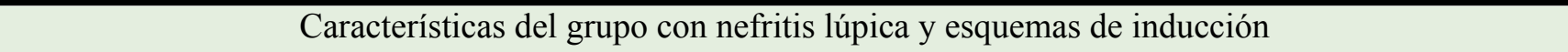 } \\
\hline Número & Género & Edad & Clase & $\begin{array}{l}\text { SIntomas no } \\
\text { renales }\end{array}$ & $\begin{array}{c}\text { Fecha de } \\
\text { ingreso }\end{array}$ & Inducción & Mantenimiento \\
\hline 1 & $\mathrm{~F}$ & 36 & ND & $\mathrm{MC}, \mathrm{ME}$ & $22 / 04 / 10$ & $\begin{array}{l}\text { MF-P: Remisión parcial con recaída } \\
\text { NIH }(03-13,04-13,05-13,07-13,08-13,11- \\
\text { 13): No respuesta }\end{array}$ & MF,P \\
\hline 2 & M & 26 & 111 & $\mathrm{ME}$ & $\begin{array}{l}10 / 01 / 13 \\
23 / 02 / 10\end{array}$ & P - H: Remisión completa & $\mathrm{P}, \mathrm{H}$ \\
\hline 3 & M & 33 & IV & $\mathrm{MC}, \mathrm{G}$ & & $\begin{array}{l}\text { MF-P: Remisión parcial con recaída } \\
\text { NIH (07-12, 08-12, 09-12, 10-12, 11-12, 12- } \\
\text { 12): Remisión parcial con recaída } \\
\text { R (12-13): Remisión parcial }\end{array}$ & MF,P,H \\
\hline 4 & M & 40 & 111 & $\mathrm{MC}, \mathrm{ME}$ & 03/02/09 & $\begin{array}{l}\text { MF-P-H: Remisión parcial con recaída } \\
\text { Euro-lupus } 2012 \text { (09-07, 24-07, 14-08, 29- } \\
\text { 08, 12-09, 27-09): Remisión completa }\end{array}$ & $\mathrm{AZ}, \mathrm{D}, \mathrm{H}$ \\
\hline 5 & $\mathrm{~F}$ & 25 & IV & $\mathrm{CR}, \mathrm{H}$ & 01/03/11 & MF-P: Remisión completa & - \\
\hline 6 & $\mathrm{~F}$ & 55 & IV & - & $22 / 09 / 09$ & AZ-D-H: Remisión completa & $\mathrm{AZ}, \mathrm{D}, \mathrm{H}$ \\
\hline 7 & $\mathrm{~F}$ & 67 & II & - & $24 / 03 / 09$ & AZ-P-H: Remisión completa & $\mathrm{H}$ \\
\hline 8 & $\mathrm{~F}$ & 27 & I & $\mathrm{CR}$ & $04 / 08 / 12$ & AZ-D-H: Remisión completa & $\mathrm{D}, \mathrm{H}$ \\
\hline 9 & $\mathrm{~F}$ & 29 & III & $\mathrm{MC}$ & $10 / 11 / 13$ & $\begin{array}{l}\text { NIH (11-13, 12-13, 01-14, 02-14): Pendiente } \\
2 \text { dosis }\end{array}$ & \\
\hline 10 & M & 34 & II & $\mathrm{MC}, \mathrm{ME}$ & $18 / 12 / 13$ & IECA y ARA II & \\
\hline 11 & $\mathrm{~F}$ & 28 & IV & $\mathrm{CR}, \mathrm{MC}$ & $29 / 10 / 12$ & $\begin{array}{l}\text { NIH (11-12, 12-12, 01-13, 02-13, 03-13, 04- } \\
\text { 13): Remisión completa AZ,D,H }\end{array}$ & \\
\hline 12 & $\mathrm{~F}$ & 31 & ND & $\mathrm{MC}, \mathrm{CR}, \mathrm{H}$ & $05 / 07 / 11$ & MF-P-H: Remisión completa & MF,P,H \\
\hline 13 & M & 24 & III & ME & $25 / 02 / 09$ & $\begin{array}{l}\text { Euro-lupus (9 dosis en 2009): Remisión } \\
\text { parcial con recaída } \\
\text { R (01-11): Remisión parcial con recaída } \\
\text { NIH (02-13, 03-13, 04-13, 05-13, 06-13, 07-13): } \\
\text { Remisión parcial con recaída }\end{array}$ & MF,P,H \\
\hline 14 & $\mathrm{~F}$ & 44 & IV & ME & 28/01/09 & MF-D-H: Remisión completa & MF,D \\
\hline 15 & M & 30 & II & G & $06 / 05 / 12$ & $\begin{array}{l}\text { MF-P: Remisión parcial con recaída } \\
\text { NIH }(09-13,10-13,11-13,12-13,01-14,02- \\
\text { 14): Remisión parcial }\end{array}$ & $\mathrm{MF}, \mathrm{P}, \mathrm{H}$ \\
\hline 16 & $\mathrm{~F}$ & 43 & III & $\mathrm{ME}$ & $16 / 03 / 09$ & AZ - H: Remisión completa & $\mathrm{AZ}, \mathrm{H}$ \\
\hline 17 & M & 18 & IV & $\mathrm{G}, \mathrm{CR}$ & $18 / 12 / 12$ & $\begin{array}{l}\text { NIH (02-13, 03-13, 04-13, 05-13, 06-13, 06- } \\
\text { 13): Remisión completa }\end{array}$ & MF,D,H \\
\hline 18 & $\mathrm{~F}$ & 35 & IV & $\mathrm{MC}, \mathrm{CR}$ & $30 / 05 / 12$ & $\begin{array}{l}\text { Euro-lupus } 2012 \text { (18-08, 03-09, 13-09, } \\
28-09,12-10,29-10) \text { : Remisión parcial con } \\
\text { recaida } \\
\text { MF-D-H: Remisión parcial }\end{array}$ & $\mathrm{MF}, \mathrm{D}, \mathrm{H}$ \\
\hline 19 & M & 23 & IV & $\mathrm{G}, \mathrm{MC}$ & $25 / 11 / 10$ & MF-D-H: Remisión parcial & MF,D,H \\
\hline
\end{tabular}

G: Generales, MC: Mucocutáneos, ME: Musculoesqueléticos, CR: Cardiorespiratorios, H: Hematológicos. MF: Micofenolato Mofetilo, P: Prednisona, H: Hidroxicloroquina, AZ: Azatioprina, D: Deflazacort, IECA: Inhibidores de la enzima convertidora de angiotensina, ARA II: Antagonistas de los receptores de angiotensina. 
de las muestras. La proteinuria basal promedio fue de $2480 \mathrm{mg} / 24$ horas (91-6640 mg/24horas). Los pacientes con proteinuria en rango nefrótico corresponden a $37 \%$ de la población (Tabla 2).

La proporción de anticuerpos antiDNA positivos fue de $64 \%$ y de hipocomplementemia C3 de $71 \%$ y C4 de $57 \%$.

El valor promedio de leucocitos fue de $8500 / \mathrm{mm}^{3}$, de hemoglobina fue $13.1 \mathrm{~g} / \mathrm{dL}$ y de plaquetas $263362 /$ $\mathrm{mm}^{3}$.

Al término del seguimiento se encontró que la creatinina promedio fue de $1.00 \mathrm{mg} / \mathrm{dL}(0.5-2.59 \mathrm{mg} / \mathrm{dL})$ y la tasa de FGe (CKD-EPI) promedio de $91.86 \mathrm{ml} /$ $\mathrm{min} / 1.73 \mathrm{~m} 2(20.05-116.03 \mathrm{ml} / \mathrm{min} / 1.73 \mathrm{~m} 2)$. En el $13 \%(\sigma=3.33)$ de la población se presentó deterioro en la tasa de FGe. La proteinuria promedio fue de $1470 \mathrm{mg} / 24$ horas (51.5-1955 mg/24 horas) con disminución de la proteinuria en el $75 \%(\sigma=3843)$ de la población analizada.

14 pacientes $(87.5 \%)$ lograron algún tipo de remisión a los 6 meses, de los cuales 8 pacientes $(50 \%)$ lograron remisión completa y $6(37.5 \%)$ remisión parcial. Entre los pacientes con remisión completa no se encontró recaída durante el seguimiento (mediana 70 meses). En 5 pacientes con remisión parcial se encontró evidencia de recaída durante el seguimiento (mediana 120 meses). No se encontraron diferencias entre los pacientes tratados con ciclofosfamida y con micofenolato mofetil. Entre los pacientes con NL proliferativa, el $41.6 \%$ tuvo inducción con esquema NIH, el $25 \%$ con esquema Euro-lupus y en el $5.5 \%$ de los pacientes se realizó la inducción con micofenolato mofetil y prednisolona (Tabla 1). No se encontró asociación entre el uso del esquema $\mathrm{NIH}$ y la respuesta clínica total o parcial $(\mathrm{p}=0.09)$. No se encontró asociación entre el esquema NIH y la disminución en proteinuria $(p=0.2)$. No se presentó asociación entre la utilización de micofenolato mofetil y la respuesta clínica total o parcial $(\mathrm{p}=0.14)$.

\section{Discusión}

La nefritis lúpica (NL) fue reconocida, por primera vez, por Sir William Osler (1895) como parte del

Tabla 2

Características clínicas de la población con nefritis lúpica.

\begin{tabular}{|c|c|c|c|c|c|c|}
\hline Característica & Media & Desviación Estandar & Media & $\begin{array}{c}\text { Error } \\
\text { Estandar }\end{array}$ & Mínimo & Máximo \\
\hline Edad (años) & 34,9 & 12,7 & 32,0 & 3,2 & 18 & 67,0 \\
\hline SEXO & \multicolumn{6}{|c|}{7 Hombres $(43 \%)$ y 9 Mujeres $(57 \%)$} \\
\hline Creatinina (mg/dl) & 1,2 & 1,1 & 0,9 & 0,3 & 0,5 & 5,0 \\
\hline $\mathrm{TFG}(\mathrm{ml} / \mathrm{min} / 1.73 \mathrm{~m} 2)$ & 93,4 & 39,4 & 101,7 & 9,8 & 11,0 & 148,1 \\
\hline BUN (mg/dl) & 18,7 & 10,2 & 18,5 & 2,6 & 7,5 & 45,5 \\
\hline Leucocitos & 8606,9 & 4418,8 & 7105,0 & 1104,7 & 4690,0 & 21790,0 \\
\hline Hemoglobina $(\mathrm{g} / \mathrm{dl})$ & 13,2 & 1,8 & 13,4 & 0,5 & 10,0 & 17,1 \\
\hline Plaquetas & 265688 & 68913 & 257000 & 17228 & 83000 & 363000 \\
\hline Densidad urinaria & 1,0 & 0,0 & 1,0 & 0,0 & 1,0 & 1,0 \\
\hline $\mathrm{pH}$ urinario & 5,8 & 0,6 & 6,0 & 0,2 & 5,0 & 6,5 \\
\hline Hematuria (N /campo) & 17,6 & 24,5 & 12,0 & 6,1 & 0,0 & 100,0 \\
\hline Proteinuria 24 h (mg) & 2205,9 & 1845,3 & 1745,0 & 461,3 & 91,0 & 6640,0 \\
\hline $\mathrm{C} 3(\mathrm{mg} / \mathrm{dl})$ & 64,6 & 33,5 & 51,8 & 9,0 & 15,0 & 133,0 \\
\hline $\mathrm{C} 4(\mathrm{mg} / \mathrm{dl})$ & 11,5 & 8,6 & 9,0 & 2,3 & 1,7 & 24,1 \\
\hline
\end{tabular}

Distribución de las variables clínicas de la población analizada. TFG: Tasa de filtración glomerular estimada por fórmula MDRD4. BUN: Nitrógeno ureico. C3: Complemento C3, C4: Complemento C4. 
LES, pero fue sólo a partir de 1940 cuando se conoció ampliamente su fisiopatología. Desde entonces ha existido un cambio en la historia natural del compromiso renal por LES. El pronóstico a largo plazo se modificó favorablemente gracias al uso de los corticoides introducidos en la década de los $60 \mathrm{y}$, posteriormente, a los agentes citostáticos y citotóxicos que disminuyen la actividad de la enfermedad.

El pronóstico y respuesta al tratamiento dependen del patrón inicial histológico, teniendo mejor pronóstico las lesiones limitadas al mesangio (Tipo I y II) y de peor pronóstico las variantes proliferativas (Tipo III y IV). Altos índices de cronicidad limitan la respuesta terapéutica mientras que los criterios de actividad son marcadores de progresión inflamatoria y demandan inicio de esquema terapéutico prontamente. El comportamiento rápidamente progresivo definido como la pérdida de más del $50 \%$ de la función renal en un periodo menor a 3 meses o la presencia de más del $50 \%$ de crecentes en la biopsia renal, es otro de los marcadores de progresión crítica de la enfermedad y demanda una acción rápida y oportuna $^{7,8}$.

La respuesta a la terapia en los pacientes intervenidos oportunamente no siempre es inmediata, en los primeros meses de inducción la tasa de remisión parcial es de alrededor del $80 \%$, evidenciándose disminución de la proteinuria y estabilización de los azoados. Razón por la cual las terapias de mantenimiento deben sostenerse por periodos superiores a 1 año luego de alcanzar la remisión.

En la actualidad, se define como remisión completa a los pacientes que reducen la proteinuria a $<0.3$ $\mathrm{g} / \mathrm{d}$ o relación proteinuria creatinina $\mathrm{Pru} / \mathrm{CrS}:<0.2$, hematuria $<10 x c$, enfermedad extrarrenal inactiva y normalización de pruebas serológicas. Se considera remisión parcial si la proteinuria disminuye por debajo de $1 \mathrm{~g} /$ día $^{10}$.

Desde la introducción de los esteroides por Polak en 1964, hasta la inmunosupresión moderna se ha mejorado la supervivencia global y el periodo libre de diálisis de los pacientes con NL. En la actualidad la terapia inicial de la NL está basada en los hallazgos de la biopsia renal, encontrándose entre los factores predictores de buen pronóstico, a largo plazo, la proteinuria $<1 \mathrm{~g} / 24$ horas y la disminución de la creatinina sérica. La remisión en los primeros 6 meses se asocia con mayor sobrevida libre de diálisis y menor exposición a inmunosupresores ${ }^{11,12}$.

En nuestros pacientes, el 75\% presentó disminución de proteinuria y el $87.5 \%$ alcanzó algún tipo de remisión. Es relevante tener en cuenta que en el 37\% de los pacientes se encontró proteinuria en rango nefrótico, cuando claramente es fundamental el inicio de la terapia de modo temprano.

Desde la década de los 80 los pulsos de ciclosfosfamida asociados a esteroides mostraron mejoría de sobrevida renal y remisión de la enfermedad, convirtiéndose en el tratamiento estándar. Los buenos resultados de los esquemas combinados y dosis bajas de inmunosupresores se han traducido en una menor toxicidad derivada de la terapia; por lo cual en el presente siglo se introdujo como parte de las herramientas terapéuticas el micofenolato, agente con menores efectos colaterales y respaldado en los estudios de Chan, Ginzler, Contreras, el ALMS (Aspreva Lupus Management Study) y 2 metaanálisis del 2007, mostrando no ser inferior a los esquemas basados en ciclofosfamida y en algunos de estos experimentos los resultados clínicos fueron levemente superiores $^{13-20}$. En nuestro estudio no encontramos diferencias en la respuesta a los diferentes esquemas de inducción (ciclofosfamida o micofenolato) con respecto a los tipos histológicos estudiados, tipo de remisión alcanzada, ni otros desenlaces, este hallazgo es concordante con los resultados de los estudios previamente mencionados.

\section{Conclusiones}

Entre los pacientes con nefritis lúpica proliferativa del Hospital Militar Central de Bogotá, Colombia, se encuentran similares porcentajes de remisión y recaídas a lo descrito en la población general. No encontramos diferencia con respecto al porcentaje de remisión, disminución de creatinina y proteinuria cuando comparamos los esquemas de inducción basados en ciclofosfamida con micofenolato.

\section{Conflicto de interés:}

Los autores declaran no tener ningún conflicto de interés. 


\section{Bibliografía}

1. Kdigo Clinical Practice Guideline For glomerulonephritis. Chapter 12: Lupus nephritis. Kidney International Supplements 2012;2:221-232.

2. Gourley M, Austin H, Scott D, et al. Metthylprednisolone and Cyclophosphamide, alone or in combination in patients with Lupus Nephritis. Ann InternMed 1996;125:549-557.

3. Hossiau FA, Vasconcelos C, DCruz D, et al. Immuno suppressive therapy in lupus nephritis: The Eurolupus. Arthritis Rheum 2002;46:2121-2131.

4. Isenberg D, Appel G, Contreras. Et al. Influence of race/ethnicity on response to lupus nephritis treatment. Rheumatology 2010;49:128-140.

5. Anaya J, Cañas C, Mantilla R. Lupus nephritis in Colombians, contrasts an comparison with other population. Clinical Reviews in Allergy \& Immunology 2011;40:199-207.

6. Ramsey-Goldman R, Isenberg D. Systemic Lupus Erythematosus Measures Arthritis \& Rheumatism 2003; 49 (5S): S225-S233.

7. Mok CC. Prognostic factors in lupus nephritis. Lupus 2005;14:39-44.

8. Pinto Peñaranda LF, Castro Mercado IL, Duque Caballero V, et al. Predictive risk factors for failure to induction therapy of lupus nephritis in a cohort of Colombian patients. ReumatolClin. 2014;10(3):147-51.

9. Clark W, Sontrop J. What have we learned about optimal induction therapy for lupus nephitis (III through V) form Randomized controlled trials? Clin J Am SocNephrol 2008;3:895-898.

10. Bertsias G, Ioannidis J et al. EULAR recommendations for the management of systemic lupus erythematosus. Report of a Task Force of the EULAR Standing Committee for International Clinical Studies Including Therapeutics. Ann Rheum Dis 2008:67;195-205.

11. Barber CE, Geldenhuys L, Hanly JG. Sustained remission of lupus nephritis. Lupus. 2006;15(2):94-101.

12. Contreras G, Pardo V, Cely C, et al. Factors associated with poor outcomes in patients with lupus nephritis. Lupus 2005;14(11):890-5.

13. Chan T, Eung F, et al. Efficacy of mycophenolatemofetil in patients with diffuse proliferative lupus nephritis. $\mathrm{N}$ Eng $\mathrm{J}$ Med 2000;343:1156-62.

14. Chan T, Tse K. Long term study of mycophenatemofetil as continuous induction and maintenance treatment for diffuse proliferative lupus nephritis. J Am SocNephrol 2005;16:1076-1084.

15. Ginzler E, Dooley M, et al. MycophenolateMofetil or Intravenous Cyclophosphamide for Lupus Nephritis. N Engl J Med 2005;353:2219-28.

16. Contreras G, Pardo V, et al. Sequential Therapies for Proliferative Lupus Nephritis. N Engl J Med 2004;350:971-80.

17. Sinclair A, Appel G, et al. Mycophenolatemofetil as induction and maintenance therapy for lupus nephritis: rationale and protocol for the randomized, controlled Aspreva Lupus Management Study (ALMS). Lupus 2007;16:972-980.

18. ONG L, HOOI L, et al. Randomized controlled trial of pulse intravenous cyclophosphamide versus mycophenolatemofetil in the induction therapy of proliferative lupus nephritis. Nephrol 2005;10:504-510.

19. Walsh M, James M, et al. MycophenolateMofetil for Induction Therapy of Lupus Nephritis: A Systematic Review and Meta-Analysis. Clin J Am SocNephrol 2007;2:968-975.

20. Zhu B, Chen N. Mycophenolatemofetil in induction and maintenance therapy of severe lupus nephritis: a meta-analysis of randomized controlled trials. Nephrol Dial Transplant 2007;22:1933-1942. 